\title{
RADICAL SURGERY FOR INVASIVE VAGINAL CARCINOMA
}

\author{
N. Bacalbașa ${ }^{1}$, Irina Bălescu², V. Brașoveanu ${ }^{3}$ \\ 1"Carol Davila” University of Medicine and Pharmacy, Bucharest, Romania \\ 2“"Ponderas" Hospital, Bucharest, Romania \\ ${ }^{3}$ Department of General Surgery and liver Transplantation "Dan Setlacec" \\ Fundeni Clinical Institute, Bucharest, Romania
}

\begin{abstract}
Vaginal cancer represents a rare malignancy accounting for $2 \%$ of all gynecologic malignant tumors. Due to its rarety, there are no prospective trials to guide the treatment. The most commonly used treatment option is radiotherapy. We present the case of a 75 years old female who was addressed to our service for vaginal bleeding. The local exam showed the presence of a large tumor on the posterior vaginal wall and the biopsies revealed squamous cell carcinoma. The patient was refferred to the radiotherapy service but after 5 sequences of external beam therapy associated with chemotherapy (Cisplatin) she developped an episode of severe rectal bleeding so the radiation therapy was cancelled. We perfoemed a total colpectomy with total hysterectomy, bilateral adnexectomy and bilateral pelvic and inguinal lymph node dissection. The histopathological findings confirmed the preoperative results of the biopsies. In the postoperative course the patient developped an episode of severe diarheea and dehidratation due to the irradiation recto-colitis which needed intensive hidroelectrolitic re-equilibration. The patient was discharged the 20th postoperative day. 2 years postoperatively there are no signs of recurrent disease.
\end{abstract}

Keywords: invasive vaginal squamous carcinoma, radiotherapy, recto-colitis, total colpectomy, lymph node dissection

\section{Introduction}

Primary vaginal carcinoma is a rare malignant tumor constituting up to $2 \%$ of all malignant gynecologic tumors $(1,2,3)$. Almost $80 \%$ of vaginal carcinoma are metastatic from the cervix, endometrium or vulva. $(1,2)$ Any primary neoplasia involving both the vaginal wall and the cervix is considered cervical cancer and treated as such; any tumor involving both vagina and vulvar region is considered vulvar cancer. All other vaginal tumors with no expression on cervix or vulva are considered primary vaginal malignancy. (1)
Biopsies of the tumor are then performed in order to establish the histopathological type; the most common findings are squamous cell carcinoma, clear cell carcinoma, melanoma and sarcoma. In cases when squamous cell carcinoma is diagnosed, radiation therapy associated with chemosensitization with weekly cisplatin is the main treatment. $(2,3,4)$ In patients who develop severe complications due to radiotherapy an individualized treatment is needed. In this case we were compelled to interrupt the oncologic treatment and decision to switch to radical surgery was made. 


\section{Case report}

A 75 year old patient was addressed to our service for severe vaginal bleeding. The local exam showed a vaginal tumor developed in the middle third of the posterior wall. (fig. 1) Biopsies were taken and a squamous cell non-keratinized carcinoma was found. Immunohistochemistry studies revealed CK34beta 12 positive, P16 - negative, P53 - nega-tive, Ki67 - positive in $45 \%$ of the tumoral cells. An MRI was performed in order to establish the local invasion and the right staging of the disease: the 45/22/28 mm tumor invaded the right levator ani muscle but did not infiltrated the rectal wall; infra-centimetric pelvic and inguinal adenopathies were also found; no distant lesions were described. The patient was confined to the oncology service for radiation therapy associated with cisplatin chemo-sensitization but after 5 sequences she developed severe recto-colitis and rectal bleeding. The onco-logic treatment was stopped and after an intense pre-operative reequilibration we performed a total colpectomy en bloc with total hysterectomy and bilateral adnexectomy. (figs. 2, 3) Bilateral pelvic and inguinal lymph node dissection was also performed. (figs. 4,5,6,7,8) In the $7^{\text {th }}$ postoperative day the patient developped an episode of severe diarheea and dehidratation. The coprological examination did not find and infectious causes; the severe symptomatology was considered to be secondary to the irradiation recto-colitis and needed intensive hidro-electrolitic re-equilibration. The patient was discharged the $20^{\text {th }}$ postoperative day. 2 years postoperatively there are no signs of recurrent disease.

\section{Discussions}

The vaginal structures, opposite to the other gynecologic tissues rarely present malignant transformations, most of the cases occurring in elderly women.(2) Squamous cell carcinoma is the most common histological type; due to the thin vaginal wall, squamous cell carcinoma usually invades the surrounding organs including the rectal wall and the urinary bladder. The lymphatic route is another important pattern of dissemination which has been described in up to $29 \%$ of patients (20 din 1); there are two main routes of lymphatic dissemination: for the upper and middle third of the vagina in pelvic obturatory nodes, iliac, and para-aortic nodes and

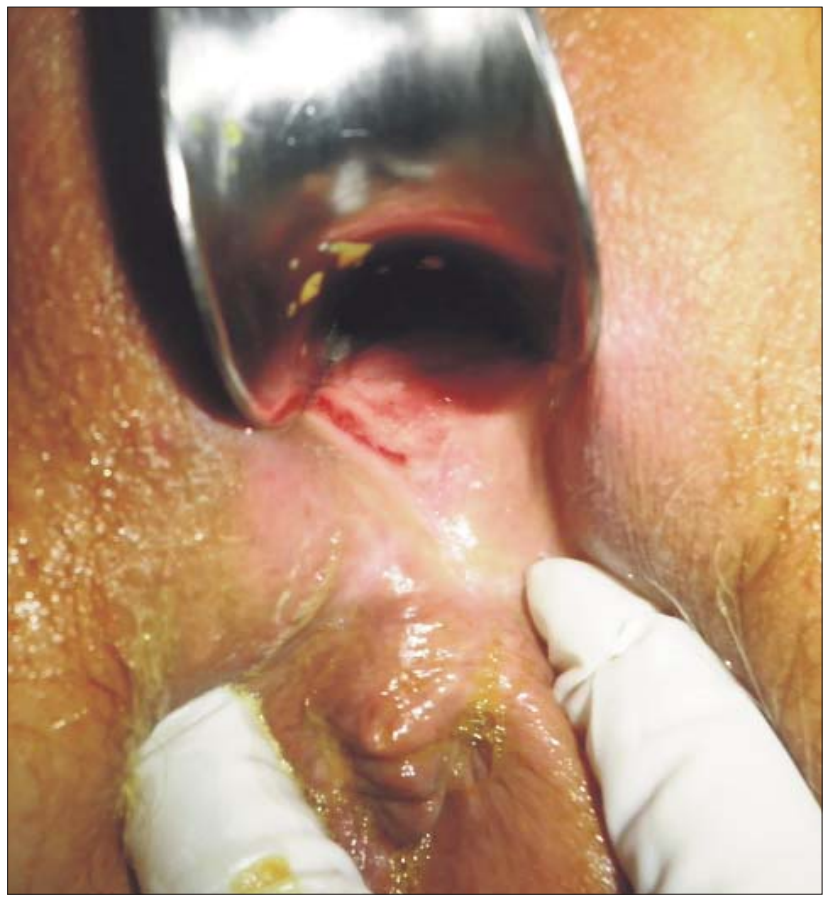

Figure 1 - The aspect of the vaginal tumor after 5 sequences of radiation therapy

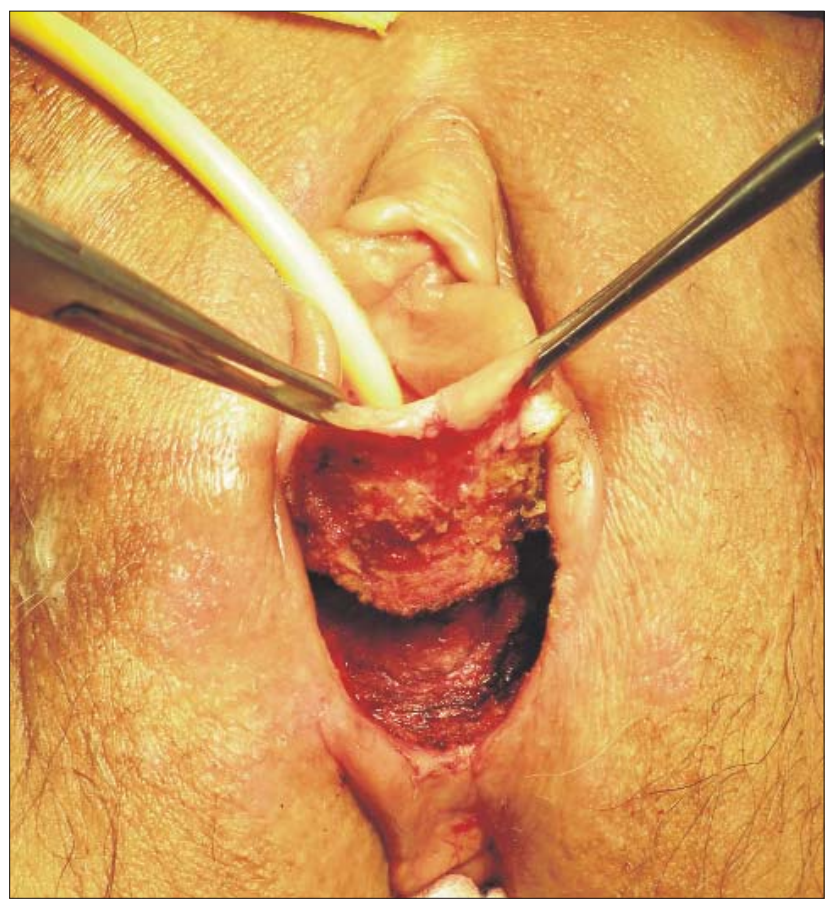

Figure 2 - Dissection of the posterior vaginal wall

for the lower third in the inguinal and then pelvic nodes $(1,2)$

Because of the rarity of this pathology there are few retrospective studies which analyze the outcomes of different approaches $(5,6)$; most 


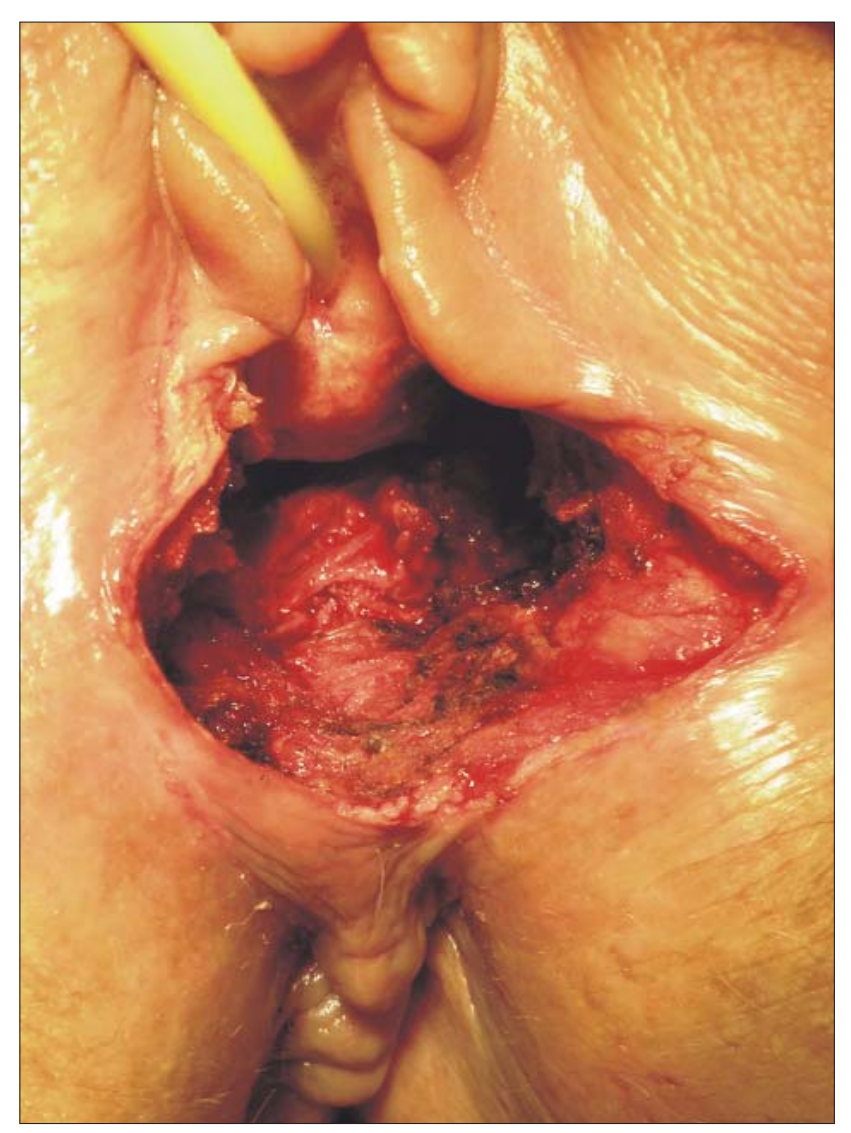

Figure 3 - The final aspect after total colpectomy

treatment guides have been adopted from more common malignancies such as cervical or anal neoplasia (7). Since 1999 when the National Cancer Institute proved the benefit of combining chemosensitization therapy -Cisplatin - with radiation therapy, this became one of the most important guidelines of treatment (8). However there are cases in which severe adverse effects occur and the radiotherapy must be stopped; the most important side effects are rectal bleeding, rectal stenosis, rectovaginal fistulas (2).

In these cases, surgical treatment is needed. Other indications for surgery are stage I disease and stage IVa disease especially if rectovaginal or vesicovaginal fistulas are found, recurrences after radiotherapy and other aggressive histological types like melanoma or leiomiosarcoma. (1) In stage IVa cases an exenterative procedure is to be performed. In the other cases (like in our patient) when radiation therapy can not be continued a radical hysterectomy with total clopectomy is performed. The goal of the radical hysterectomy in such cases is to remove the adjacent tissues of

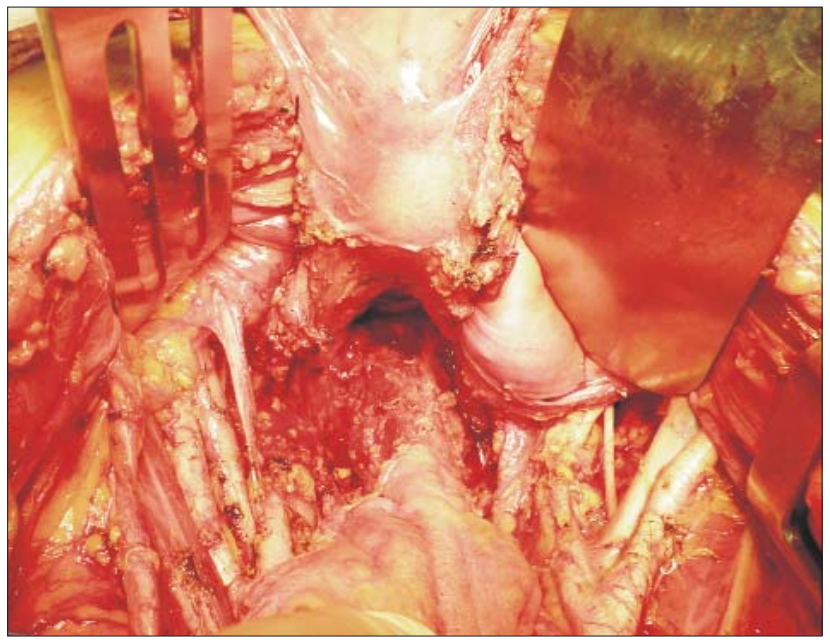

Figure 4 - The final aspect of the pelvic lymph node dissection

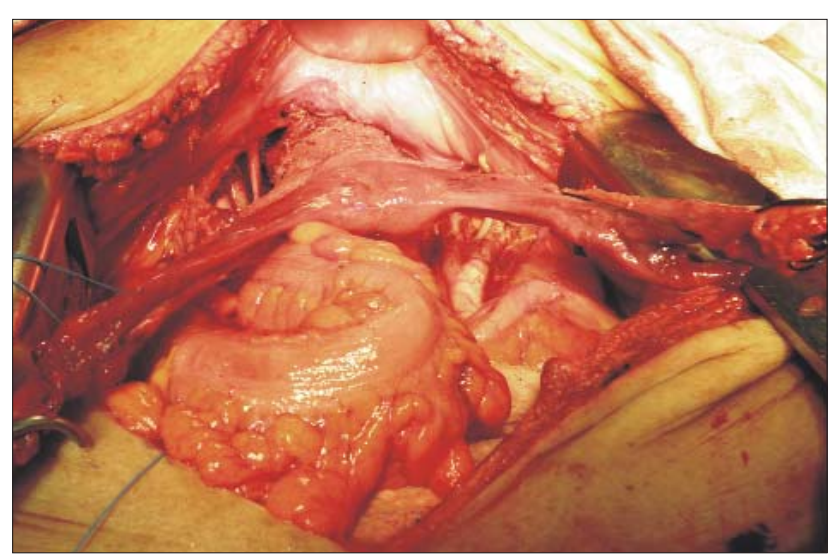

Figure 5 - The uterus and the 2 adnexa are completely mobilized

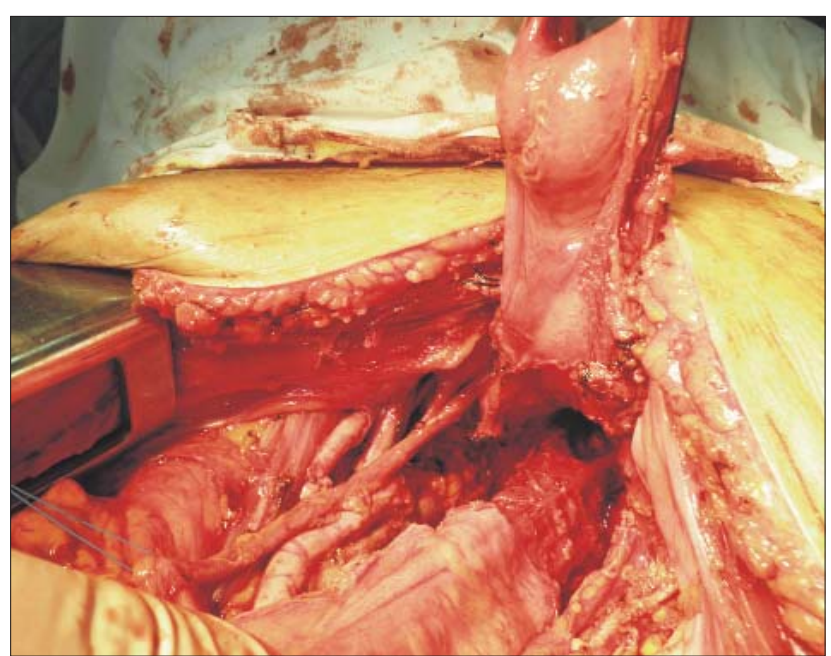

Figure 6 - The final aspect before removing the specimen. The ureters are also completely dissected 


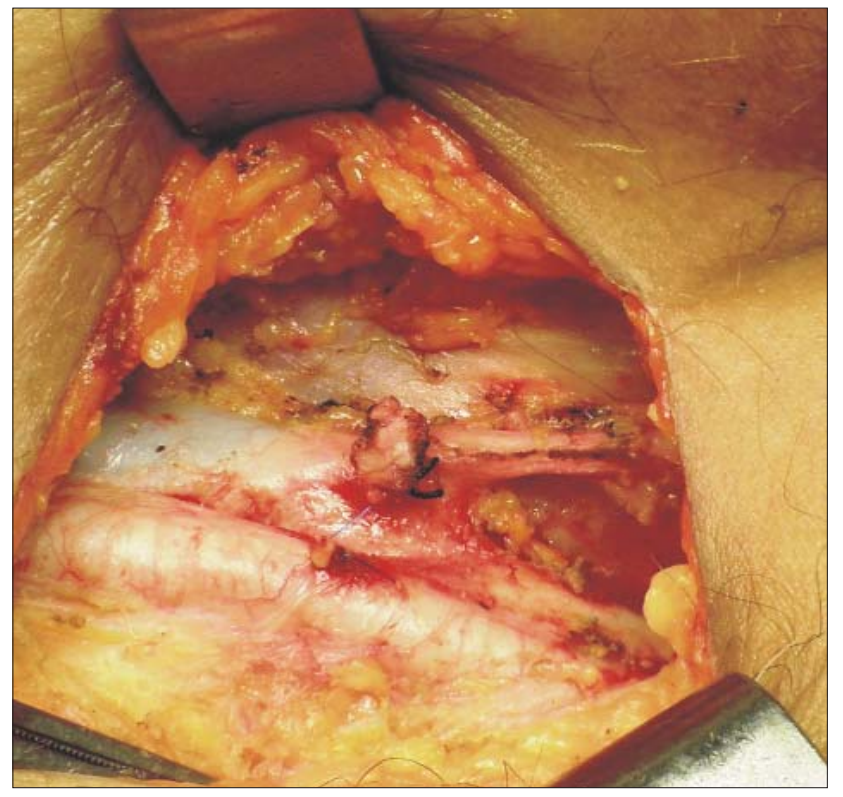

Figure 7 - The inguinal lymph node dissection - final aspect

the cervix and vaginal fornices en bloc with uterine and vagina removing, while preserving a functional urinary bladder and rectum. (1)

The most important clinical and pathological factors that affect survival include the size of the tumor ( $>3 \mathrm{~cm}$ is a sign of poor prognosis), the lateral extension of the tumor, the histopathological type (squamous cell carcinoma has a better prognosis when compared to malignant melanoma or leiomiosarcoma) and the presence of positive lymph nodes $(1,2,9,10)$. Morley et al reported a 5 year rate of survival of $70 \%$ in patients with invasive vaginal carcinoma and negative lymph nodes and $0 \%$ in cases with positive lymph nodes (9). Age can also affect the postoperative mortality rate but not the 5 year survival rate. Matthews reported a higher rate of postoperative mortality in elderly patients (>65 years old). (11)

In our case the histopathological type of the tumor was squamous cell carcinoma with no lymph node metastases; the lateral invasion in the right levator ani muscle disappeared at the moment of the surgical procedure due to the preoperative oncological treatment. The postoperative course was influenced by the reaparition of inflammatory bowel disease as a side effect of radiation therapy but it was successfully treated in a conservative manner. Grade 3 or 4 toxicity of

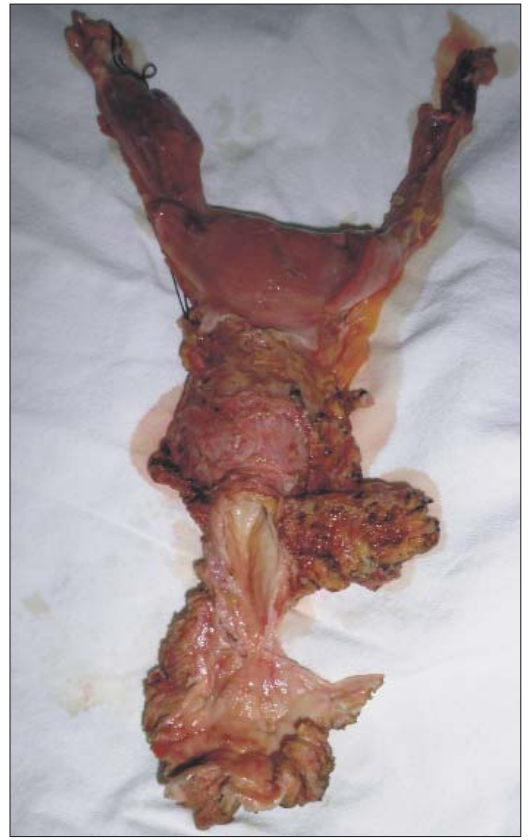

Figure 8 - The specimen: total colpectomy en bloc with total hysterectomy and bilateral adnexectomy

radiation therapy include rectovaginal fistula, proctitis, colitis, small bowell obstruction and affects especially the patient treated with external beam radiation therapy or brachyterapy (10 up $20 \%$ ). A better result concerning the toxicity is given by the intensity modulated radiotherapy (IMRT) - up to $0 \%$ grade 3 or 4 toxicity but its' role in the treatment of vaginal cancer is not well established. In Hiniker's study up to $20 \%$ of patients treated with IMRT alone developed distant recurrence (3).

\section{Conclusions}

Radical surgery for invasive vaginal carcinoma is addressed for patients with locally advanced disease (stage IVa); for the other cases radiation therapy is to be proposed. If the patient presents important complications as rectovaginal fistulas, rectal bleeding, small bowel obstruction, the radiation therapy should be stopped and a radical surgery should be considered. In our case, a radical hysterectomy en bloc with total colpectomy and bilateral inguinal and pelvic lymph node dissection offered a good control of the disease, the patient presenting no signs of local or distant recurrence at a 2 years follow-up. 


\section{References}

1. Bardawil, T., Manetta, A., Huh, W., Vaginal Cancer, emedicine medscape. com/article/ 269188 -overview\#aw2aab6c14

2. Slomovitz, B., Coleman, R., Invasive Cancer of the Vagina, Clinical Gynecologic Oncology (Eighth Edition)

3. Hiniker, S., Roux, A., Murphy, J., Primary squamous cell carcinoma of the vagina: Prognostic factors, treatment patterns and outcomes, Gynecologic Oncology 131 (2013) 380-385

4. Nashiro T, Yagi C, Hirakawa M, Inamine M, Nagai Y, Sakumoto $\mathrm{K}$, et al. Concurrent chemoradiation for locally advanced squamous cell carcinoma of the vagina: case series and literature review. Int J Clin Oncol 2008;13:335-9

5. Perez CA, Grigsby PW, Garipagaoglu M, Mutch DG, Lockett MA. Factors affecting long-term outcome of irradiation in carcinoma of the vagina. Int J Radiat Oncol Biol Phys 1999;44:37-45.
6. Frank SJ, Jhingran A, Levenback C, Eifel PJ. Definitive radiation therapy for squamous cell carcinoma of the vagina. Int $\mathrm{J}$ Radiat Oncol Biol Phys 2005;62:138-47.

7. Gray HJ. Advances in vulvar and vaginal cancer treatment. Gynecol Oncol. Jul 2010;118(1):3-5. [Medline].

8. U.S. Department of health and Human service, National Institutes of Health, National cancer Institute. $\mathrm{NCl}$ clinical announcement. Bethesda (MD): National cancer Institute; 1999.

9. Morley GW, Hopkins MP, Lindenauer SM. Pelvic exenteration, University of Michigan: 100 patients at 5 years. Obstet Gynecol. Dec 1989;74(6):934-43. [Medline].

10. Anthopoulos AP, Manetta A, Larson JE. Pelvic exenteration: a morbidity and mortality analysis of a seven-year experience. Gynecol Oncol. 1989;35(2):219-23. [Medline].

11. Matthews CM, Morris M, Burke TW. Pelvic exenteration in the elderly patient. Obstet Gynecol. May 1992;79(5 ( Pt 1)):7737. [Medline]. 